\title{
Special Toeplitz operators on strongly pseudoconvex domains
}

\section{Željko Čučković and Jeffery D. McNeal}

\begin{abstract}
Toeplitz operators on strongly pseudoconvex domains in $\mathbb{C}^{n}$, constructed from the Bergman projection and with symbol equal to a positive power of the distance to the boundary, are considered. The mapping properties of these operators on $L^{p}$, as the power of the distance varies, are established.
\end{abstract}

\section{Introduction}

Let $Y \subset X$ be Hilbert spaces and assume $X$ is also an algebra. Let $P$ : $X \longrightarrow Y$ be an orthogonal projection operator from $X$ to $Y$. If $f \in X$ is a fixed element of $X$, the Toeplitz operator with symbol $f$ associated to $P$ is the operator $T_{f}: X \longrightarrow Y$ defined by $T_{f}(g)=P(f \cdot g)$.

If $\Omega \subset \mathbb{C}^{n}$ is a bounded domain and $H(\Omega)$ denotes the holomorphic functions on $\Omega$, the Bergman projection, $B$, which orthogonally projects $L^{2}(\Omega)$ onto $A^{2}(\Omega)=: H(\Omega) \cap L^{2}(\Omega)$, is a projection operator of fundamental importance in complex analysis. One use of the Bergman projection is to show the existence of holomorphic functions on $\Omega$ which have certain additional properties, for example growth conditions measured with respect to some functional norm. In order to do this, of course, the mapping properties of the Bergman projection with respect to these additional side properties must be understood. For a general domain $\Omega$, the Bergman projection operator is completely abstract and poorly understood, but there are large classes of domains where considerable information is known.

We focus on one such class of domains here: the strongly pseudoconvex domains (the definition is in Section 3). For this class of domains, the mapping properties of $B$ in many classical Banach spaces have been established

2000 Mathematics Subject Classification: 32A36, 47B35, 32T15.

Keywords: Bergman kernel, Toeplitz operators, strongly pseudoconvex domains. 
-e.g., the Lebesgue spaces $L^{p}[16]$, the Hölder spaces $\Lambda^{\alpha}[1]-$ all as consequences of the precise information on the Bergman kernel established by Fefferman [7] on strongly pseudoconvex domains. A common feature of these mapping results is that $B$ preserves the Banach spaces in question. The preservation of Banach norms by $B$ is the best possible result one can expect: the space $A^{2}(\Omega)$ contains functions which are sharply in the various Banach spaces and $B$ leaves the space $A^{2}(\Omega)$ invariant.

In many applications, however, one needs an operator which creates holomorphic functions and whose output "improves" the global norm behavior, in some sense, of the input. A crucial feature of the Bergman kernel on a strongly pseudoconvex domain (and, indeed, on any finite type domain) is that its singularities occur only on the boundary diagonal, instead of on the full diagonal in $\Omega \times \Omega$. This suggests that a Toeplitz operator, built from $B$, with symbol equal to a positive power of $\delta_{b \Omega}=$ the distance to the boundary, $b \Omega$, of $\Omega$, will be an operator which has better "smoothing" behavior than $B$.

We call such operators distance-symbol Toeplitzoperators. Thus a distancesymbol Toeplitz operator, on a domain $\Omega \subset \mathbb{C}^{N}$ has the form

$$
T_{\delta^{\eta}}(g)(z)=: T_{\eta}(g)(z)=\int_{\Omega} B(z, w) \delta(w)^{\eta} g(w) d V(w)
$$

for some power $\eta>0$, where $B(z, w)$ denotes the Bergman kernel function associated to $\Omega$. The main result of this paper is to show how the power of the distance in the symbol of $T_{\eta}$ in (1.1) is related to the mapping properties of $T_{\eta}$ in the Lebesgue classes $L^{p}$.

Theorem 1.2. Let $\Omega \subset \subset \mathbb{C}^{N}$ be a smoothly bounded, strongly pseudoconvex domain.

(a) For $0 \leq \eta<N+1$, let $E=\frac{N+1}{N+1-\eta}$. The distance-symbol Toeplitz operator $T_{\eta}$ of the form (1.1) has the following mapping properties:

(i) If $1<p<\infty$ and $E<\frac{p}{p-1}$, then

$$
T_{\eta}: L^{p}(\Omega) \longrightarrow L^{p+G}(\Omega), \quad \text { where } G=\frac{p^{2}}{\frac{N+1}{\eta}-p}
$$

boundedly. Note that $G>0$ if $\eta>0$.

(ii) If $1<p<\infty$ and $E \geq \frac{p}{p-1}$, then

$$
T_{\eta}: L^{p}(\Omega) \longrightarrow L^{s}(\Omega) \quad \text { for any } s<\infty
$$

boundedly.

(b) For $\eta \geq N+1$, the operator of the form (1.1) maps $L^{1}(\Omega) \longrightarrow L^{\infty}(\Omega)$ boundedly. 
Most work on Bergman-Toeplitz operators usually considers holomorphic (or harmonic) symbols and poses questions involving algebraic properties of the operators or preservation of Hardy or Bergman classes of functions. Our result focuses on Bergman-Toeplitz operators as fractional integral operators, very much in the spirit of Hardy-Littlewood and Sobolev, and addresses how the size of $\eta$ effects the "smoothing" properties of these operators on the entire $L^{p}$ class of functions. Additionally, previous work on Bergman-Toeplitz operators have primarily dealt with cases where an explicit formula for the Bergman kernel is known, for example on euclidean balls in $\mathbb{C}^{n}$. It seems that the type of results in Theorem 1.2, especially in the absence of an explicit formula for the Bergman kernel, have not been considered before.

However, experts will recognize that our proof of Theorem 1.2 goes through, with minimal changes, on other classes of domains where good estimates on the Bergman kernel are known, e.g. finite type domains in $\mathbb{C}^{2}$, convex domains of finite type in $\mathbb{C}^{N}$. It is also clear that further results can be obtained by substituting for $L^{p}$ other Banach spaces - e.g. the Hölder spaces $\Lambda^{\alpha}$, the Sobolev spaces $L_{s}^{p}$ - though this would require somewhat more complicated functional analysis than we use for the $L^{p}$ spaces. Our justification for presenting only Theorem 1.2 in this paper is that the hypotheses (and conclusions) allow us to show the simple size estimates which are involved in any result of this type, with minimal geometric notation and functional analytic setup. Our methods are very simple, and they are closely related to widely used techniques, cf. [18, Chapter 7] and [16], but they also contain some modifications of these techniques.

We also want to emphasize that our theorem concerns only Toeplitz operators built from the Bergman projection. For other projection operators, e.g. the Szegö projection, there is no indication that the type of result in Theorem 1.2 will occur for any corresponding Toeplitz operator. The singularities of the Szegö kernel, for example, are spread throughout the diagonal of the region where the kernel is integrated; thus, there is no positive function, analogous to $\delta_{b \Omega}$ in Theorem 1.2, which could serve as the symbol of a Szegö-Toeplitz operator and reduce the singularity of the Szegö kernel.

The paper is laid out as follows. In Section 2 we prove some general functional analysis results about operators from $L^{p}$ to $L^{s}$, in Section 3 we recall the estimates on the Bergman kernel we shall need and prove Theorem 1.2, and in Section 4 we examine the sharpness of the estimates stated in Theorem 1.2.

Acknowledgement. The authors thank the referee for his careful reading of this paper and, especially, for his suggestions for improving Section 4. The sharpness results in Section 4 were significantly extended in the range of $p$ because of the suggestions made by the referee. 


\section{Kernels which "gain" in Lebesgue classes}

In this section, we prove two results about the $L^{p} \longrightarrow L^{s}$ mapping properties of general integral operators. These theorems simply account for how integrability conditions on the kernel of an integral operator imply integrability conditions on the output of the operator.

Results of this type are widely used and well-known, for instance Young's inequality [9] and Schur's lemma [17]. However, the authors were unable to find Proposition 2.1 and Proposition 2.8 in the literature and so feel obliged to provide their (simple) proofs. As with Young's inequality and Schur's lemma, our proofs are elementary and use only Hölder's inequality. Indeed, our statements reduce to Young's inequality and Schur's lemma when $p=s$.

The first result concerns integral operators on a general measure space.

Proposition 2.1. Let $(M, \mu)$ be a $\sigma$-finite measure space. Suppose that $K$ is a measurable function on $M \times M$, and consider the integral operator

$$
O f(x)=\int_{M} K(x, y) f(y) d \mu(y)
$$

(a) Let $1<p<\infty$ and $1 \leq s<\infty$. Let $q$ be the conjugate exponent of $p, \frac{1}{p}+\frac{1}{q}=1$ and suppose that for some $0 \leq t \leq 1$,

$$
\sup _{x} \int_{M}|K(x, y)|^{t q} d \mu(y)=C_{t, q}<\infty
$$

and

$$
\sup _{y} \int_{M}|K(x, y)|^{(1-t) s} d \mu(x)=C_{t, s}<\infty .
$$

Then (2.2) defines a bounded linear operator $O: L^{p}(M) \longrightarrow L^{s}(M)$ and $\|O\|_{o p} \leq C_{t, q}^{\frac{1}{q}} \cdot C_{t, s}^{\frac{1}{s}}$.

(b) Suppose that $1<p<\infty$ and

$$
\sup _{x} \int_{M}|K(x, y)|^{q} d \mu(y)=C_{1, q}<\infty .
$$

Then (2.2) defines a bounded linear operator $O: L^{p}(M) \longrightarrow L^{\infty}(M)$ and $\|O\|_{o p} \leq C_{1, q}^{\frac{1}{q}}$.

(c) Suppose that

$$
\sup _{x, y}|K(x, y)|=C_{\infty, \infty}<\infty .
$$

Then (2.2) defines a bounded linear operator $O: L^{1}(M) \longrightarrow L^{\infty}(M)$ and $\|O\|_{o p} \leq C_{\infty, \infty}$. 
Proof. (a) From (2.3) and Hölder's inequality, we obtain

$$
\begin{aligned}
|O f(x)| & =\left|\int_{M} K^{1-t}(x, y) f(y) K^{t}(x, y) d \mu(y)\right| \\
& \leq\left(\int_{M}|K(x, y)|^{(1-t) p}|f(y)|^{p} d \mu(y)\right)^{1 / p} \cdot C_{t, q}^{\frac{1}{q}} .
\end{aligned}
$$

Thus

$$
|O f(x)|^{s} \leq C_{t, q}^{\frac{s}{q}} \cdot\left(\int_{M}|K(x, y)|^{(1-t) p}|f(y)|^{p} d \mu(y)\right)^{s / p}
$$

which implies

$$
\begin{aligned}
\left(\int_{M}|O f(x)|^{s} d \mu(x)\right)^{p / s} \\
\leq C_{t, q}^{\frac{p}{q}}\left(\int_{M}\left(\int_{M}|K(x, y)|^{(1-t) p}|f(y)|^{p} d \mu(y)\right)^{s / p} d \mu(x)\right)^{p / s} \\
\quad \leq C_{t, q}^{\frac{p}{q}} \int_{M}\left(\int_{M}|K(x, y)|^{(1-t) s}|f(y)|^{s} d \mu(x)\right)^{p / s} d \mu(y) \\
\leq C_{t, q}^{\frac{p}{q}} \cdot C_{t, s}^{\frac{p}{s}} \int_{M}|f(y)|^{p} d \mu(y) .
\end{aligned}
$$

The second inequality in (2.7) follows from Minkowski's integral inequality, see for example [8, page 194], and the third inequality follows from (2.4). The statements of (a) all follow from inequality (2.7).

(b) Hölder's inequality directly gives

$$
|O f(x)| \leq\left(\int_{M}|K(x, y)|^{q} d \mu(y)\right)^{1 / q} \cdot\|f\|_{p},
$$

from which the conclusions of (b) follows.

(c) In this case, one simply notes that

$$
\sup _{x}|O f(x)| \leq C_{\infty, \infty} \int_{M}|f(y)| d \mu(y),
$$

and from this the conclusions of (c) follow.

There are several parameters present in the statement of Proposition 2.1, and these can be manipulated in various ways, depending on the problem at hand. In this paper, the theorem will be used as follows: given a kernel 
$K(x, y)$ and a given integrability class $L^{p}$, we shall seek the largest $s, s>p$, such that both (2.3) and (2.4) hold for some $t, 0 \leq t \leq 1$. We seek the largest such $s$ since our measure spaces will be $L^{p}$ classes of functions on bounded domains in euclidean space with Lebesgue measure (thus, $\mu(X)<\infty$ ), and we are trying to understand the "smoothing" properties of the operator associated to $K(x, y)$. Clearly, larger $s$ in Proposition 2.1 means better mapping behavior in this case. In a different direction, notice that if $p=s$, then choosing $t=\frac{1}{q}$ in Proposition 2.1 causes (2.3) and (2.4) to become the hypotheses for Young's inequality.

The second result concerns integral operators defined on $L^{p}$ classes of functions on smoothly bounded domains $D$ in $\mathbb{R}^{N}$, and is a direct extension of Proposition 2.1 to these type of operators. The point of this extension is most easily illustrated by an example. Let $D \subset \subset \mathbb{R}^{2}$ be a smoothly bounded domain in the plane and consider the kernel

$$
K(x, y)=\left(\delta_{b D}(x)+\delta_{b D}(y)+|x-y|\right)^{-2}, \quad x, y \in D .
$$

Let $1<p<\infty$ and $q$ be the conjugate exponent of $p$. The best result that Proposition 2.1 implies is that the operator

$$
O f(x)=\int_{D} K(x, y) f(y) d y
$$

maps $L^{p} \longrightarrow L^{s}$ for any $s<p$. This is so because (2.3) holds only for $t<\frac{1}{q}$, which forces $1-t>\frac{1}{p}$, and, thus, $s$ must be strictly less than $p$ in order that (2.4) holds. For this simple operator though, one expects that $O$ actually maps $L^{p}$ to $L^{p}$ boundedly. Notice that if one takes $t=\frac{1}{q}$ and $s=p$, the left hand sides of (2.3) and (2.4) just barely fail to be finite: the best bounds on the integrals blow up like the logarithm of the distance of $x$ or $y$ to the boundary of $D$, respectively. The hypotheses of Proposition 2.2 below give substitute inequalities to (2.3) and (2.4) which do allow one to conclude the "endpoint" mapping property that was expected.

We formulate the theorem for domains in $\mathbb{C}^{N}$, because of our applications in the next section, but note that the complex structure plays no role in the actual result.

Proposition 2.8. Let $\Omega \subset \subset \mathbb{C}^{N}$ be a smoothly bounded domain defined by the real-valued function $r$, i.e. $\Omega=\{z: r(z)<0\}$ and $d r \neq 0$ when $r=0$.

Let $1<p<\infty$ be given and let $q$ be the conjugate exponent of $p, \frac{1}{p}+\frac{1}{q}=1$. Also, let $1<s<\infty$.

Suppose that $K$ is a measurable function on $\Omega \times \Omega$ and, for some $0 \leq$ $t \leq 1$, and for all small $\epsilon>0$

$$
\int_{\Omega}|K(x, y)|^{t q}|r(y)|^{-\epsilon} d y \leq C_{t, q} \cdot|r(x)|^{-\epsilon}
$$


and

$$
\int_{\Omega}|K(x, y)|^{(1-t) s}|r(x)|^{-\epsilon} d x \leq C_{t, s} \cdot|r(y)|^{-\epsilon} .
$$

Then, for any $f \in L^{p}(\Omega)$, the function

$$
O f(x)=\int_{\Omega} K(x, y) f(y) d y
$$

belongs to $L^{s}(\Omega)$. Here dy denotes integration, in the $y$ variables, with respect to the euclidean volume form. Moreover, the operator $O: L^{p}(\Omega) \longrightarrow L^{s}(\Omega)$ is a bounded linear operator and $\|O\|_{o p} \leq C_{t, q}^{\frac{1}{q}} \cdot C_{t, s}^{\frac{1}{s}}$.

Proof. The proof is quite similar to the proof of Proposition 2.1. From (2.9) and Hölder's inequality, we obtain, for small $\delta>0$,

$$
\begin{aligned}
& |O f(x)|=\left.\left|\int_{\Omega} K^{1-t}(x, y)\right| r(y)\right|^{\delta} f(y) K^{t}(x, y)|r(y)|^{-\delta} d y \mid \\
& \leq\left(\int_{\Omega}|K(x, y)|^{(1-t) p}|f(y)|^{p}|r(y)|^{p \delta} d y\right)^{1 / p}\left(\int_{\Omega}|K(x, y)|^{t q}|r(y)|^{-q \delta} d y\right)^{1 / q} \\
& \leq\left(\int_{\Omega}|K(x, y)|^{(1-t) p}|f(y)|^{p}|r(y)|^{p \delta} d y\right)^{1 / p} C_{t, q}^{\frac{1}{q}} \cdot|r(x)|^{-\delta} \\
& \leq C_{t, q}^{\frac{1}{q}}\left(\int_{\Omega}|K(x, y)|^{(1-t) p}|f(y)|^{p}|r(y)|^{p \delta}|r(x)|^{-p \delta} d y\right)^{1 / p} .
\end{aligned}
$$

Thus Minkowski's inequality and (2.10) yield

$$
\begin{aligned}
& \left(\int_{\Omega}|O f(x)|^{s} d(x)\right)^{p / s} \\
& \leq C_{t, q}^{\frac{p}{q}}\left(\int_{\Omega}\left(\int_{\Omega}|K(x, y)|^{(1-t) p}|f(y)|^{p}|r(y)|^{p \delta}|r(x)|^{-p \delta} d y\right)^{s / p} d x\right)^{p / s} \\
& \leq C_{t, q}^{\frac{p}{q}} \int_{\Omega}\left(\int_{\Omega}|K(x, y)|^{(1-t) s}|f(y)|^{s}|r(y)|^{s \delta}|r(x)|^{-s \delta} d x\right)^{p / s} d y \\
& =C_{t, q}^{\frac{p}{q}} \int_{\Omega}|f(y)|^{p}|r(y)|^{p \delta}\left(\int_{\Omega}|K(x, y)|^{(1-t) s}|r(x)|^{-s \delta} d x\right)^{p / s} d y \\
& \leq C_{t, q}^{\frac{p}{q}} \cdot C_{t, s}^{\frac{p}{s}} \int_{\Omega}|f(y)|^{p}|r(y)|^{p \delta}|r(y)|^{-p \delta} d y \\
& =C_{t, q}^{\frac{p}{q}} C_{t, s}^{\frac{p}{s}}|| f \|_{p}^{p} .
\end{aligned}
$$

The desired conclusions follow from (2.12). 
We point out that the defining function $r$ is not the only function which could be used in the proof above. Other auxiliary functions, suited to the problem at hand, could replace $r$ in (2.9) and (2.10); the cancellation of powers of this auxiliary function would still occur in the penultimate line of (2.12), resulting in the conclusion stated in Proposition 2.8. The formulation of (a version of) Proposition 2.8 on more general manifolds with boundary is similarly straightforward.

\section{Estimates on the Bergman kernel}

Let $\Omega \subset \mathbb{C}^{N}$ be a domain, and let $H(\Omega)$ denote the holomorphic functions on $\Omega$. The Bergman projection, $B$, is the orthogonal projection operator $B: L^{2}(\Omega) \longrightarrow H(\Omega)$, where $L^{2}(\Omega)$ is standard space of square-integrable functions with respect to Lebesgue measure and orthogonality is meant in the sense of the standard $L^{2}$ inner product. The Bergman kernel function, $B(z, w) \in H(\Omega) \times \overline{H(\Omega)}$, represents the Bergman projection as an integral operator

$$
B f(z)=\int_{\Omega} B(z, w) f(w) d w, \quad f \in L^{2}(\Omega),
$$

where $d w$ denotes integration in the $w$ variables, with respect to the euclidean volume form. The Bergman kernel function is a highly domain dependent function, whose behavior at the boundary of $\Omega$ is not known, in general.

Suppose that $\Omega$ is smoothly bounded, that is: there exists a $C^{\infty}$, realvalued function $r: \operatorname{nbhd}(\bar{\Omega}) \longrightarrow \mathbb{R}$ such that $\Omega=\{z: r(z)<0\}$ and $d r \neq 0$ when $r=0$. If $\Omega$ is strongly pseudoconvex, i.e. $i \partial \bar{\partial} r(p)(\xi, \bar{\xi})>0$ for all $p \in b \Omega$ and all nonzero vectors $\xi \in \mathbb{C}^{N}$ satisfying $\partial r(p)(\xi)=0$, the boundary behavior of the Bergman kernel function associated to $\Omega$ is understood quite precisely. In fact, Fefferman [7] has established a complete asymptotic expansion of $B(z, w)$, in terms of $r(z), r(w)$ and a pseudo-distance between $z$ and $w$, as $z, w \rightarrow b \Omega$ (see also [2]). We do not need the precision of an asymptotic expansion here, and so we recall only the (essentially sharp) upper bounds on the Bergman kernel.

Proposition 3.1. Let $\Omega=\{r<0\}$ be a smooth, bounded, strongly pseudoconvex domain in $\mathbb{C}^{N}$. For each $p \in b \Omega$, there exists a neighborhood $U$ of $p$, holomorphic coordinates $\left(\zeta_{1}, \ldots, \zeta_{N}\right)$ and a constant $C>0$, such that for $a, b \in U \cap \Omega$

$$
|B(a, b)| \leq C\left(|r(a)|+|r(b)|+\left|a_{1}-b_{1}\right|+\sum_{k=2}^{N}\left|a_{k}-b_{k}\right|^{2}\right)^{-(N+1)} .
$$

Here $a=\left(a_{1}, \ldots, a_{N}\right)$ in the $\zeta$-coordinates, and similarly for $b$. 
The earlier works of [10] and [5] were important precursors to Fefferman's result; however the results there contained only estimates on the Bergman kernel restricted to the diagonal, which are not sufficient for obtaining mapping properties of the Bergman projection. Inequality (3.2) can be extracted from the results in [7]; it may also be obtained by simpler methods than those used by Fefferman. The simplest known derivation of Proposition 3.1 uses the scaling methods developed in [12] and [15], see the remark (5.3) in [12]. For more details on this proof, see [13].

It is elementary to see that a defining function is uniformly comparable to $\delta_{b \Omega}$ : there exist constants $c_{1}, c_{2}$, which depend on $r$ but not on $z \in \Omega$, such that $c_{1} \delta_{b \Omega}(z) \leq|r(z)| \leq c_{2} \delta_{b \Omega}(z)$ for all $z \in \Omega$ (implicit function theorem). So, for our distance-symbol Toeplitz operators, we may consider the operators

$$
\begin{aligned}
\mathcal{T}_{\eta} f(z) & =B\left(|r|^{\eta} f\right)(z)=\int_{\Omega} B(z, w)|r(w)|^{\eta} f(w) d w \\
& =\int_{\Omega} K_{\eta}(z, w) f(w) d w
\end{aligned}
$$

for any defining function $r$, establish mapping properties of the operators $\mathcal{T}_{\eta}$, then infer the same properties on the operators $T_{\eta}$.

In order to apply Proposition 2.8, we examine the size of powers of $K_{\eta}$ integrated against $|r|^{-\epsilon}$. To avoid writing irrelevant constants below, we use the notation $f(z) \lesssim g(z)$ to denote that there exists a constant $C$, independent of $z$, such that $f(z) \leq C g(z)$.

Proposition 3.4. Let $\Omega=\{r<0\}$ be a smooth, bounded, strongly pseudoconvex domain in $\mathbb{C}^{N}$, and let $B(z, w)=B_{\Omega}(z, w)$ be the Bergman kernel associated to $\Omega$. then

If $K_{\eta}(z, w)=B(z, w)|r|^{\eta}(w)$, if $0 \leq \eta<N+1$, and if $\rho \leq \frac{N+1}{N+1-\eta}$,

$$
\int_{\Omega}\left|K_{\eta}(z, w)\right|^{\rho}|r(w)|^{-\epsilon} d w \lesssim|r(z)|^{-\epsilon}
$$

for all $0<\epsilon<1$.

Proof. The proof follows the lines of Lemma 1 in [14]. Let $\Delta_{b}=\{(z, z)$ : $z \in b \Omega\}$ be the boundary diagonal of $\bar{\Omega} \times \bar{\Omega}$. We recall that

$$
B(z, w) \in C^{\infty}\left(\bar{\Omega} \times \bar{\Omega} \backslash \Delta_{b}\right),
$$

see $[11]$. 
Cover $b \Omega$ by neighborhoods $U_{1}, \ldots U_{M}$ given by Proposition 3.1 ; we may assume that the neighborhoods are so small that the quantity in parenthesis on the right hand side of (3.2) is less than 1. If $U_{0}=\bar{\Omega} \backslash \cup_{j=1}^{M} U_{j}$ then, it follows from (3.6) that $\int_{U_{0}}\left|K_{\eta}(z, w)\right|^{\rho}|r(w)|^{-\epsilon} d w \lesssim 1$, if $z \in \bar{\Omega}$.

Now consider an arbitrary $U_{j}, 1 \leq j \leq M$, and work in the coordinates associated to $U_{j}$ by Proposition 3.1. It follows from (3.2) that if $a, b \in \bar{\Omega} \cap U_{j}$,

$$
\left|K_{\eta}(a, b)\right| \lesssim\left(|r(a)|+|r(b)|+\left|a_{1}-b_{1}\right|+\sum_{k=2}^{N}\left|a_{k}-b_{k}\right|^{2}\right)^{-(N+1)+\eta} .
$$

Let $a \in U_{j}$ be temporarily fixed. If $\rho \leq \frac{N+1}{N+1-\eta}$, it follows from (3.7) that

$$
\begin{aligned}
I_{j} & =\int_{U_{j}}\left|K_{\eta}(a, b)\right|^{\rho}|r(b)|^{-\epsilon} d b \\
& \lesssim \int_{\mathbb{C}^{N}}\left(|r(a)|+|r(w)|+\left|a_{1}-w_{1}\right|+\sum_{k=2}^{N}\left|a_{k}-w_{k}\right|^{2}\right)^{\rho(-(N+1)+\eta)}|r(w)|^{-\epsilon} d w \\
& \lesssim \int_{\mathbb{C}^{N}}\left(|r(a)|+|r(w)|+\left|a_{1}-w_{1}\right|+\sum_{k=2}^{N}\left|a_{k}-w_{k}\right|^{2}\right)^{-(N+1)}|r(w)|^{-\epsilon} d w .
\end{aligned}
$$

Now change coordinates: $\tilde{w}_{k}=w_{k}-a_{k}, k=2, \ldots, N$, Re $\tilde{w}_{1}=r(w)$, $\operatorname{Im} \tilde{w}_{1}=\operatorname{Im} w_{1}$. And to simplify notation, let $x=\operatorname{Re} \tilde{w}_{1}$ and $y=\operatorname{Im} a_{1}-$ $\operatorname{Im} w_{1}$. We then obtain

$$
I_{j} \lesssim \int_{\mathbb{C}^{N}}\left(|r(a)|+|x|+|y|+\sum_{k=2}^{N}\left|\tilde{w}_{k}\right|^{2}\right)^{-(N+1)}|x|^{-\epsilon} d \tilde{w}_{2} \ldots d \tilde{w}_{N} d x d y
$$

First consider the $\tilde{w}_{2}$ integration in (3.8). Define

$$
\begin{aligned}
& R_{1}=\left\{\tilde{w}_{2}:\left|\tilde{w}_{2}\right|^{2}>|r(a)|+|x|+|y|+\sum_{k=3}^{N}\left|\tilde{w}_{k}\right|^{2}\right\} \\
& R_{2}=\left\{\tilde{w}_{2}:\left|\tilde{w}_{2}\right|^{2}<|r(a)|+|x|+|y|+\sum_{k=3}^{N}\left|\tilde{w}_{k}\right|^{2}\right\} .
\end{aligned}
$$

Using polar coordinates on the region $R_{1}$ we have

$$
\begin{gathered}
\int_{R_{1}}\left(|r(a)|+|x|+|y|+\sum_{k=2}^{N}\left|\tilde{w}_{k}\right|^{2}\right)^{-(N+1)}|x|^{-\epsilon} d \tilde{w}_{2} \leq \int_{R_{1}}\left(\left|\tilde{w}_{2}\right|^{2}\right)^{-(N+1)}|x|^{-\epsilon} d \tilde{w}_{2} \\
\lesssim \int_{L}^{\infty} R^{-2(N+1)} R|x|^{-\epsilon} d R \lesssim\left(|r(a)|+|x|+|y|+\sum_{k=3}^{N}\left|\tilde{w}_{k}\right|^{2}\right)^{-N}|x|^{-\epsilon},
\end{gathered}
$$


where $L=\left(|r(a)|+|x|+|y|+\sum_{k=3}^{N}\left|\tilde{w}_{k}\right|^{2}\right)^{1 / 2}$. However, on the region $R_{2}$ we obtain the same upper bound by elementary estimation:

$$
\begin{aligned}
& \int_{R_{2}}\left(|r(a)|+|x|+|y|+\sum_{k=2}^{N}\left|\tilde{w}_{k}\right|^{2}\right)^{-(N+1)}|x|^{-\epsilon} d \tilde{w}_{2} \\
& \quad \leq\left(|r(a)|+|x|+|y|+\sum_{k=3}^{N}\left|\tilde{w}_{k}\right|^{2}\right)^{-(N+1)}|x|^{-\epsilon} \operatorname{vol}\left(R_{2}\right) \\
& \quad \lesssim\left(|r(a)|+|x|+|y|+\sum_{k=3}^{N}\left|\tilde{w}_{k}\right|^{2}\right)^{-N}|x|^{-\epsilon} .
\end{aligned}
$$

The same type of estimation may be performed on the $d \tilde{w}_{3}, \ldots d \tilde{w}_{N}$ integrals, reducing one negative power of the integrand at each step, to obtain

$$
I_{j} \lesssim \int_{\mathbb{C}}(|r(a)|+|x|+|y|)^{-2}|x|^{-\epsilon} d x d y
$$

For this final integral, estimate the $y$ integration first, in the same manner as above. Then, noting that the extra power of $-\epsilon$ for the $x$ integral avoids the occurrence of a logarithm term, we obtain as above that,

$$
I_{j} \lesssim|r(a)|^{-\epsilon}, \quad a \in \Omega \cap U_{j} .
$$

Since we obtain the identical bounds on each of the open sets $U_{1}, \ldots, U_{M}$, and since $I_{j} \lesssim 1$ if $z \notin U_{j}$ by (3.6), we have shown (3.5).

We are now prepared to give the

Proof of Theorem 1.2. First consider the case where $\eta \geq N+1$. It follows directly from (3.2) and (3.6) that

$$
\sup _{z, w \in \bar{\Omega}}\left|K_{\eta}(z, w)\right| \lesssim 1
$$

Part (c) of Proposition 2.1 thus implies that $\mathcal{T}_{\eta}: L^{1}(\Omega) \longrightarrow L^{\infty}(\Omega)$ boundedly, so (b) of Theorem 1.2 holds.

Next consider the case where $\eta<N+1$. Let $1<p<\infty$ and suppose that $E\left(=\frac{N+1}{N+1-\eta}\right) \geq \frac{p}{p-1}$. Then Proposition 3.4 says that

$$
\int_{\Omega}\left|K_{\eta}(z, w)\right|^{q}|r(w)|^{-\epsilon} d w \lesssim|r(z)|^{-\epsilon},
$$

so (2.9) holds for any $t<1$. Taking $t$ arbitrarily close to 1 , we then see that (2.10) holds for any $s<\infty$ by Proposition 3.4. Thus, (a) part (ii) of Theorem 1.2 holds, by Proposition 2.8. 
Finally, in the case where the hypotheses of (a) part (i) of Theorem 1.2 are satisfied, it follows from Proposition 3.4 that (2.9) and (2.10) will hold for an $s, 1<s<\infty$, if we can find a $t, 0 \leq t \leq 1$ such that both

$$
t \frac{p}{p-1} \leq E \quad \text { and } \quad(1-t) s \leq E,
$$

hold. Making the first inequality in (3.9) an equality defines the largest possible $t$ for the first inequality to hold; substituting this value of $t$ into the second inequality then gives the stated relationship on $s$ in Theorem 1.2 (a) part (i).

\section{On sharpness of the mapping properties}

We shall consider the results of Theorem 1.2 in the special case of the Bergman spaces $A^{p}(\mathbb{D})$, for $\mathbb{D}$ the unit disc in complex plane, for $1<p<\infty$. Thus, we have $N=1$ in Theorem 1.2 and we look at the Toeplitz operator $T_{(1-|z|)^{\eta}}$.

Claim: For any $1<p<\infty$, and for any $0<\eta<\frac{2}{p}$, the operator $T_{(1-|z|)^{\eta}}$ maps $L^{p}(\mathbb{D})$ to no higher $L^{s}(\mathbb{D})$ class than that stated in Theorem 1.2 (i).

In order to show the Claim, we first collect 2 one variable facts.

Lemma 4.1. If $f \in L^{1}(\mathbb{D})$ is a real-valued, radial function, we have for all integers $k \geq 0$ that

$$
T_{f} z^{k}=c_{k} z^{k}, \quad \text { where } c_{k}=(2 k+2) \hat{f}(2 k+2) .
$$

Here

$$
\hat{f}(x)=\int_{0}^{1} f(\rho) \rho^{x-1} d \rho
$$

is the Mellin transform of $f$.

Proof. If $\left\{e_{j}\right\}=\left\{\sqrt{j+1} z^{j}\right\}, j=0,1, \ldots$ denotes the standard orthonormal basis for $A^{2}(\mathbb{D})$, then

$$
T_{f} z^{k}=\sum_{j=0}^{\infty}(j+1)\left\langle f z^{k}, z^{j}\right\rangle z^{j}
$$

It is easy to see that $\left\langle f z^{k}, z^{j}\right\rangle=\hat{f}(2 k+2)$ if $j=k$ and $\left\langle f z^{k}, z^{j}\right\rangle=0$ if $j \neq k$ (see [4] for more details on the Mellin transform and Toeplitz operators).

Suppose now that $f$ is a real-valued, bounded, radial function on $\mathbb{D}$. Since the Bergman projection $B$ is a bounded operator from $L^{p}(\mathbb{D})$ to $A^{p}(\mathbb{D})$ for 
$1<p<\infty$, it is clear that $T_{f}$ is a bounded operator from $L^{p}(\mathbb{D})$ to $A^{p}(\mathbb{D})$ for $1<p<\infty$. It is well-known that for this range of $p$, the partial sums of the Taylor series of a function in $A^{p}(\mathbb{D})$ converge, in norm, to the function. Hence, if $h(z)=\sum_{k=0}^{\infty} a_{k} z^{k} \in A^{p}(\mathbb{D})$, then $T_{f} h(z)=\sum_{k=0}^{\infty} a_{k} c_{k} z^{k}$ by Lemma 4.1. Recall that a sequence $\left\{\lambda_{k}\right\}$ is a coefficient multiplier from $A^{p}(\mathbb{D})$ to $A^{q}(\mathbb{D})$ if $\sum_{k=0}^{\infty} \lambda_{k} a_{k} z^{k} \in A^{q}(\mathbb{D})$ whenever $\sum_{k=0}^{\infty} a_{k} z^{k} \in A^{p}(\mathbb{D})$. Thus for $T_{f}$, with $f$ as above, we have that the sequence $\left\{c_{k}\right\}$ given in Lemma 4.1 is a coefficient multiplier on $A^{p}(\mathbb{D})$ to itself, for $1<p<\infty$. For an extensive treatment of coefficient multipliers of the Bergman space on the unit disc, see the recent book [6].

The second one variable result we need is the following:

Lemma 4.2. Let $f(z)=(1-|z|)^{\eta}$, for $\eta>0$, on $\mathbb{D}$. Then

$$
(2 k+2) \hat{f}(2 k+2) \sim k^{-\eta} .
$$

Proof. If $c_{k}=(2 k+2) \hat{f}(2 k+2)$, then integrating by parts repeatedly gives

$$
c_{k}=2(k+1) \int_{0}^{1}(1-\rho)^{\eta} \rho^{2 k+1} d \rho=\text { const. } \frac{(2 k+2) !}{(\eta+1) \ldots(\eta+2 k+2)} .
$$

This can be expressed using the gamma function as

$$
c_{k}=\text { const. } \frac{\Gamma(2 k+3) \Gamma(\eta+1)}{\Gamma(\eta+2 k+3)},
$$

which is asymptotic to $k^{-\eta}$, by Stirling's formula.

Remark: From the formula for $c_{k}$ above, notice that $c_{k}$ can be expressed in terms of the Beta function, instead of the Mellin transform. That is,

$$
c_{k}=(2 k+2) B(2 k+2, \eta+1) \quad \text { where } B(a, b)=\int_{0}^{1} s^{a-1}(1-s)^{b-1} d s .
$$

To prove the sharpness of our Theorem 1.2, part (i), we will use some results from [3]. We also mention the paper [19] which contains related, precursor results to those in [3]. In Theorem 1.3, part (a), of [3], it is shown that the sequence $\left\{k^{-\eta}\right\}$ multiplies $A^{p}(\mathbb{D})$ into $A^{s}(\mathbb{D})$ with $s=\frac{2 p}{2-\eta p}$. For $\eta<2 / p$ (so that $s<\infty$ ), we are then in the case of our Theorem 1.2 (i), and $s=\frac{2 p}{2-\eta p}$ is precisely the value of $s$ that our Theorem 1.2 (i) guarantees $T_{\eta}$ maps $L^{p}(\mathbb{D})$ to, in dimension 1. Since Lemma 4.2 above shows that the sequence corresponding to $T_{\eta}$ is $c_{k} \sim k^{-\eta}$, the mapping results of the two papers are consistent with each other (though not sub-Theorems of each other, for the reason mentioned in the paragraph below). 
Turning now to the question of sharpness, Lemma 3.8 in [3], and the remark following it, shows that $s=\frac{2 p}{2-\eta p}$ is the best possible integrability exponent that the sequence $\left\{k^{-\eta}\right\}$ multiplies $A^{p}(\mathbb{D})$ into. Because of Lemma 4.2 above, it is tempting to conclude immediately, therefore, that Theorem 1.2 (i) is sharp. The problem, however, is that a bounded sequence may not multiply $A^{s}(\mathbb{D})$ to itself if $s \neq 2$. (see Section 3 of [3] regarding the solid sequences and, also, Corollary 4.4). Thus, a little more work is required to connect the asymptotic statement of our Lemma 4.2 to the sharpness statements from [3].

So suppose, on the contrary, that $c_{k}=(2 k+2) B(2 k+2, \eta+1)$ does multiply $A^{p}(\mathbb{D})$ to $A^{s+\epsilon}(\mathbb{D})$, with $s=\frac{2 p}{2-\eta p}$ and $\epsilon>0$. Consider the quotient $\frac{k^{-\eta}}{c_{k}}$ :

$$
\begin{aligned}
\frac{k^{-\eta}}{c_{k}} & =\frac{1}{k^{\eta}} \cdot \frac{1}{(2 k+2) B(2 k+2, \eta+1)} \\
& =\left(\frac{2 k+1}{k}\right)^{\eta} \cdot \frac{2 k+1}{2 k+2} \cdot \frac{1}{(2 k+1)^{\eta+1} B(2 k+2, \eta+1)} \\
& =\left[2^{\eta} \frac{2 k+1}{2 k+2}\right] \cdot\left(1+\frac{1}{2 k}\right)^{\eta} \cdot \frac{1}{(2 k+1)^{\eta+1} B(2 k+2, \eta+1)}
\end{aligned}
$$

We consider the three factors in (4.3) separately. Recall that a sequence $\left\{\lambda_{k}\right\}$ is of bounded variation if $\sum_{k=0}^{\infty}\left|\lambda_{k+1}-\lambda_{k}\right|<\infty$. The first factor in (4.3) is trivially of bounded variation. The sequence in the second factor in (4.3), $\left\{\left(1+\frac{1}{2 k}\right)^{\eta}\right\}$, is also of bounded variation. To see that, apply the mean value theorem to the function $g(x)=\left(1+\frac{1}{2 x}\right)^{\eta}$ on each interval $[k, k+1]$ and estimate the derivative by $\left|g^{\prime}(x)\right| \leq \frac{1}{4} \frac{\eta}{x^{2}} \leq \frac{\eta}{k^{2}}$. By Proposition 3.7 in [3], sequences of bounded variation multiply $A^{p}(\mathbb{D})$ to itself, for $1<p<\infty$; thus both of the first two factors in (4.3) multiply $A^{s+\epsilon}(\mathbb{D})$ to itself. Finally, we consider the third factor in (4.3). The sequence $\left\{(2 k+1)^{\eta+1} B(2 k+2, \eta+1)\right\}$ is precisely the type of sequence considered in Lemma 3.4 of [3]; this lemma asserts that the sequence

$$
\left\{\left[(2 k+1)^{\eta+1} B(2 k+2, \eta+1)\right]^{-1}\right\}
$$

has the so-called Taylor form, defined on page 376 of the same paper. However, Lemma 3.2 of [3] deals with sequences of Taylor form, and it follows from this lemma that the sequence (4.4) multiplies $A^{s+\epsilon}(\mathbb{D})$ to itself.

Taken together, these facts imply that the sequence $\left\{k^{-\eta} / c_{k}\right\}$ multiplies $A^{s+\epsilon}(\mathbb{D})$ to itself. Since we are assuming that $\left\{c_{k}\right\}$ multiplies $A^{p}(\mathbb{D})$ to $A^{s+\epsilon}(\mathbb{D})$, we may compose $\left\{k^{-\eta} / c_{k}\right\}$ and $\left\{c_{k}\right\}$ and conclude that $\left\{k^{-\eta}\right\}$ multiplies $A^{p}(\mathbb{D})$ into $A^{s+\epsilon}(\mathbb{D})$ with $s=\frac{2 p}{2-\eta p}$. But this contradicts Theorem 1.3 of [3]. This contradiction proves the Claim above. 
Our results about the sharpness of the mapping properties in Theorem 1.2 are not definitive. Even for the unit disc in $\mathbb{C}$, note that we have restricted attention to small values of $\eta>0$ above $\left(0<\eta<\frac{2}{p}\right)$, i.e. the case dealt with by our Theorem 1.2 (a) (i). This is because if, for example, $\eta \geq 2$, Theorem $1.2(\mathrm{~b})$ says that $T_{\eta}$ maps $L^{1}(\mathbb{D})$ into $L^{\infty}(\mathbb{D})$ and this is tautologically sharp within the $L^{q}$ classes. But it is reasonable to expect that, as $\eta$ gets large, $T_{\eta}$ will map $L^{1}$ into better and better subclasses of $L^{\infty}$; here "better" can be interpreted in several ways, e.g. having fractional derivatives, or being in some weighted $L^{q}$ class with weights which blowup at the boundary. At least in $\mathbb{C}$, we are certain that numerous results which refine our Theorem 1.2 (b) can be obtained, by considering various subclasses of $L^{\infty}$ as the target space, but we do not have a guess about an ultimate sharpness result in this direction. More importantly, however, is our lack of knowledge about how sharp our Theorem 1.2 is for domains in $\mathbb{C}^{n}, n>1$. We have no results indicating whether the dimension dependent gain in Theorem 1.2 is sharp or simply an artifact of our proof; it may be possible that distance-symbol operators $T_{\delta_{b \Omega}}$ map $L^{p}$ to some $L^{s}$ class where $s$ is independent of the dimension. A result of this type would be quite useful in several applications.

\section{References}

[1] Ahern, P. And Schneider, R.: Holomorphic Lipschitz functions in pseudoconvex domains. Amer. J. Math. 101 (1979), 543-565.

[2] Boutet de Monvel, L. And Sjöstrand, J.: Sur la singularité des noyaux de Bergman et de Szegö. In Équations aux Dérivées Partielles de Rennes (1975), 123-164. Asterisque 34-35. Soc. Math. France, Paris, 1976.

[3] Buckley, S., Koskela, P. And Vukotić, D.: Fractional integration, differentiation, and weighted Bergman spaces. Math. Proc. Cambridge Philos. Soc. 126 (1999), 369-385.

[4] C̆uČKOvić, Z̆. AND RAO, N. V.: Mellin transform, monomial symbols, and commuting Toeplitz operators. J. Funct. Anal. 154 (1998), 195-214.

[5] Diederich, K.: Das Randverhalten der Bergmanschen Kernfunktion und Metrik in streng pseudo-konvexen Gebieten. Math. Ann. 187 (1970), 9-36.

[6] Duren, P. And Schuster, A.: Bergman spaces. Mathematical Surveys and Monographs 100. American Mathematical Society, Providence, 2004.

[7] Fefferman, C.: The Bergman kernel and biholomorphic mappings of pseudoconvex domains. Invent. Math. 26 (1974), 1-65.

[8] Folland, G.: Real Analysis. Modern techniques and their applications. Pure and Applied Mathematics. John Wiley, New York, 1984.

[9] Folland, G.: Introduction to partial differential equations. Mathematical Notes. Princeton University Press, Princeton, N.J., 1976. 
[10] Hörmander, L.: $L^{2}$ estimates and existence theorems for the $\bar{\partial}$-operator. Acta Math. 113 (1965), 89-152.

[11] Kerzman, N.: The Bergman kernel function. Differentiability at the boundary. Math. Ann. 195 (1972), 149-158.

[12] McNeal, J. D.: Boundary behavior of the Bergman kernel function in $\mathbb{C}^{2}$. Duke Math. J. 58 (1989), 499-512.

[13] McNeal, J. D.: Subelliptic estimates and scaling in the $\bar{\partial}$-Neumann problem. In Explorations in complex and Riemannian geometry, 197-217. Contemp. Math. 332. American Mathematical Society, Providence, RI, 2003.

[14] McNeal, J. D. And Stein, E. M.: Mapping properties of the Bergman projection on convex domains of finite type. Duke Math. J. 73 (1994), 177199.

[15] Nagel, A., Rosay, J.-P., Stein, E. M. and Wainger, S.: Estimates for the Bergman and Szegö kernels in $\mathbb{C}^{2}$. Ann. of Math. (2) 129 (1989), 113-149.

[16] Phong, D. H. And Stein, E. M.: Estimates for the Bergman and Szegö projections on strongly pseudo-convex domains. Duke Math. J. 44 (1977), 695-704.

[17] Saint Raymond, X.: Elementary introduction to the theory of pseudodifferential operators. Studies in Advanced Mathematics. CRC Press, Boca Raton, FL, 1991.

[18] Rudin, W.: Function theory in the unit ball of $\mathbb{C}^{n}$. Grundlehren der Mathematischen Wissenschaften 241. Springer-Verlag, New York-Berlin, 1980.

[19] Vukotić, D.: On the coefficient multipliers of Bergman spaces. J. London Math. Soc. (2) 50 (1994), 341-348.

Recibido: 18 de junio de 2004

Revisado: 30 de marzo de 2005

$$
\begin{array}{r}
\text { Željko Čučković } \\
\text { Department of Mathematics } \\
\text { University of Toledo } \\
\text { Toledo, Ohio, } 43606 \\
\text { zcukovi@math.utoledo.edu } \\
\text { Jeffery D. McNeal } \\
\text { Department of Mathematics } \\
\text { Ohio State University } \\
\text { Columbus, Ohio, } 43210 \\
\text { mcneal@math.ohio-state.edu }
\end{array}
$$

Research of the second author supported by a grant from the National Science Foundation. 